\title{
The HSP90 inhibitor 17-N-allylamino-17-demethoxy geldanamycin (17-AAG) synergizes with cisplatin and induces apoptosis in cisplatin-resistant esophageal squamous cell carcinoma cell lines via the Akt/XIAP pathway
}

\author{
TAKASHI UI ${ }^{1}$, KAZUE MORISHIMA ${ }^{1}$, SHIN SAITO ${ }^{1}$, YUJI SAKUMA ${ }^{2}$, HIROFUMI FUJII $^{3}$, \\ YOSHINORI HOSOYA ${ }^{1}$, SHUMPEI ISHIKAWA ${ }^{4}$, HIROYUKI ABURATANI ${ }^{5}$, \\ MASASHI FUKAYAMA ${ }^{6}$, TOSHIRO NIKI ${ }^{2}$ and YOSHIKAZU YASUDA ${ }^{1}$ \\ Departments of ${ }^{1}$ Surgery, ${ }^{2}$ Pathology and ${ }^{3}$ Clinical Oncology, Jichi Medical University, Shimotsuke, Tochigi 329-0498; \\ ${ }^{4}$ Division of Genomic Pathology, Medical Research Institute, Tokyo Medical and Dental University, Bunkyo-ku, \\ Tokyo 113-8510; ${ }^{5}$ Division of Genome Science, Research Center for Advanced Science and Technology, \\ the University of Tokyo, Meguro-ku, Tokyo 153-8904; ${ }^{6}$ Department of Pathology, Graduate School \\ of Medicine, the University of Tokyo, Bunkyo-ku, Tokyo 113-0033, Japan
}

Received October 9, 2013; Accepted November 20, 2013

DOI: 10.3892/or.2013.2899

\begin{abstract}
Although cisplatin (CDDP) is a key drug in the treatment of esophageal squamous cell carcinoma (ESCC), acquired chemoresistance remains a major problem. Combination therapy may represent one strategy to overcome this resistance. Heat shock protein 90 (HSP90) is known to be overexpressed in several types of cancer cells, and its inhibition by small molecules, either alone or in combination, has shown promise in the treatment of solid malignancies. In the present study, we evaluated the synergistic effects of combining CDDP with the HSP90 inhibitor 17-N-allylamino17-demethoxy geldanamycin (17-AAG) on two CDDP-resistant human esophageal squamous cancer cell lines, KYSE30 and KYSE150. The results obtained demonstrated the synergistic inhibitory effects of CDDP and 17-AAG on the growth of KYSE30 and KYSE150 cells. Cell growth and cell number were more effectively reduced by the combined treatment with CDDP and 17-AAG than by the treatment with either CDDP or 17-AAG alone. Western blotting revealed that the combined action of CDDP and 17-AAG cleaved poly (ADP-ribose) polymerase (PARP) and caspase-3, which demonstrated that the reduction in both cell growth and cell number was mediated by apoptosis. Time-course experiments showed that reduction
\end{abstract}

Correspondence to: Professor Toshiro Niki, Department of Pathology, Jichi Medical University, 3311-1 Yakushiji, Shimotsuke, Tochigi, 329-0498, Japan

E-mail: tniki@jichi.ac.jp

Key words: cisplatin, tanespimycin, HSP90 heat shock protein inhibitor, esophageal squamous cell carcinoma in X-linked inhibitor of apoptosis protein (XIAP) and phosphorylated Akt were concomitant with apoptosis. The results of the present study demonstrate that 17-AAG synergizes with CDDP and induces apoptosis in CDDP-resistant ESCC cell lines, and also that modulation of the Akt/XIAP pathway may underlie this synergistic effect. Combination therapy with CDDP and an HSP90 inhibitor may represent a promising strategy to overcome CDDP resistance in ESCC.

\section{Introduction}

Esophageal cancer is one of the most aggressive types of cancer of the gastrointestinal tract. The estimated incidence of esophageal cancer was $3.8 \%$ of all cancers and the estimated cancer mortality worldwide was $5.4 \%$ in 2008 (1). The incidence of esophageal cancer is particularly high in both males and females in East Asia, with squamous cell carcinoma being the predominant histologic type in this region, accounting for more than $90 \%$ of all esophageal cancer cases (1). Multimodality therapy, including surgery, chemotherapy and radiotherapy, is required for the effective management of advanced esophageal cancer. However, the 5-year survival rates of patients treated using surgery, chemotherapy alone, radiotherapy alone and concurrent chemoradiotherapy were reported to be $50.2,8.6,15.5$ and $26.4 \%$, respectively (2). Therefore, further improvements in outcomes are urgently required (2-5).

Cisplatin (CDDP) is a platinum drug that is widely used to treat esophageal squamous cell carcinoma (ESCC) $(6,7)$. CDDP, either alone or in combination with other agents, has been shown to improve patient outcomes. However, acquired chemoresistance develops during the course of treatment and is often the reason for treatment failure. Therefore, overcoming chemoresistance is essential for improving the outcomes of patients. 
Molecular chaperone proteins function to ensure the proper conformation of client proteins when cells experience stress or damage (8). Heat shock protein 90 (HSP90) is a molecular chaperone that participates in stabilizing and activating more than 200 proteins, including serine/threonine kinases (Akt, Raf-1, and $\mathrm{Cdk} 4$ ), and the transcription factors hypoxia-inducible factor $1 \alpha(\mathrm{HIF} 1 \alpha)$ and $\mathrm{p} 53$, receptor/nonreceptor kinases (HER2, EGFR, and Src family kinases), and steroid hormone receptors (9). Since many of these client proteins have been shown to significantly contribute to tumor growth and survival, abrogating their function with an HSP90 inhibitor is an attractive prospect (10).

In this study, we explored the potential synergistic effect of CDDP and the HSP90 inhibitor, 17-N-allylamino-17demethoxy geldanamycin (17-AAG), on human ESCC cell lines. We also attempted to identify the molecular mechanism involved in this synergistic effect.

\section{Materials and methods}

Cell lines and culture. The TE series (TE1, TE4, TE5, TE6, TE8, TE9, TE10, TE11, TE14 and TE15) and EC-GI-10 were obtained from the Riken BioResource Center (Saitama, Japan). The KYSE series (KYSE30, KYSE70, KYSE140, KYSE150, KYSE170, KYSE180, KYSE220 and KYSE270), TT, and TTn were obtained from the Health Science Foundation (Tokyo, Japan). These cell lines were derived from human esophageal squamous cancer. Cells were cultured in a $5 \% \mathrm{CO}_{2}$ atmosphere at $37^{\circ} \mathrm{C}$ in RPMI-1640 (R8758; Sigma-Aldrich, St. Louis, MO, USA) complete medium supplemented with $10 \%$ fetal bovine serum (FBS), penicillin $(100 \mathrm{U} / \mathrm{ml})$, and streptomycin $(100 \mu \mathrm{g} / \mathrm{ml})$.

Chemicals and antibodies. CDDP and 17-AAG were purchased from Sigma-Aldrich. CDDP was dissolved in $0.9 \%$ sodium chloride solution and 17-AAG was dissolved in dimethyl sulfoxide (DMSO). All drugs were stored in aliquots at $-20^{\circ} \mathrm{C}$.

Antibodies to caspase-3 (\#9662),PARP (\#9542), XIAP(3B6) (\#2045), c-IAP1 (\#4952), c-IAP2(58C7) (\#3130), livin (D61D1) XP (\#5471), survivin (71G4B7) (\#2808), phospho-Akt (Ser473) (D9E) (\#4060), Akt (\#9272), phospho-p44/42 MAPK (Erk1/2) (Thr202/Tyr204) (\#9101), and p44/p22 MAPK (Erk1/2) (\#9102) were from Cell Signaling Technologies (Beverly, MA, USA). The antibody to $\beta$-actin was from Sigma-Aldrich. Antibodies to Bcl-2 (\#610391), Bcl-xL (\#60982), Bid (\#61158), Bad (\#610391), Bax (\#610982), Beclin (\#612112), and BAG-1 (\#611868) were from BD Biosciences (San Jose, CA, USA).

Drug sensitivity assay. Cells were suspended in RPMI/10\% FBS and seeded at between 2,000 and 4,000 cells/well in quintuplicate in 96-well plates. Cells were treated with varying doses of CDDP and 17-AAG $24 \mathrm{~h}$ after plating and were allowed to grow for an additional $72 \mathrm{~h}$. Viable cell density was determined by a water-soluble tetrazolium salt (WST-8, Cell Counting kit-8; Dojindo, Japan) according to the manufacturer's instructions using a microplate absorbance reader (Bio-Rad Laboratories, Hercules, CA, USA) at $450 \mathrm{~nm}$.

Determination of $\mathrm{IC}_{50}$ and combination index $(\mathrm{CI}) . \mathrm{IC}_{50}$ was calculated using the CompuSyn software (ComboSyn, Inc.,
Paramus, NJ, USA). CI values were calculated for $50 \%$ toxicity based on the equation below (11):

$$
C I=D_{1} / D_{1}+D_{2} / D_{2}+\alpha x\left[\left(D_{1} x_{2}\right) /\left(D_{1} \times D_{2}\right)\right]
$$

where, $\mathrm{Dx}_{1}=$ Dose of drug 1 to produce $50 \%$ cell kill alone; $\mathrm{D}_{1}=$ Dose of drug 1 to produce $50 \%$ cell kill in combination with $\mathrm{D}_{2} ; \mathrm{Dx}_{2}=$ Dose of drug 2 to produce $50 \%$ cell kill alone; $\mathrm{D}_{2}=$ Dose of drug 2 to produce $50 \%$ cell kill in combination with $\mathrm{D}_{1} ; \alpha=0$ for mutually exclusive or 1 for mutually nonexclusive modes of drug action.

Time-dependent cell growth assay. Equal numbers of cells were seeded in quintuplicate in 96-well plates and cell growth was measured using WST-8 Cell Counting kit-8 at 0, 24, 48 and $72 \mathrm{~h}$. The results were expressed as percentages relative to the absorbance at $0 \mathrm{~h}$.

Western blot analysis. KYSE30 and KYSE150 cells were seeded on $100 \mathrm{~mm}$ plates and were exposed to CDDP with/without 17-AAG after $24 \mathrm{~h}$. Cells were subsequently cultured for 24, 48 and $72 \mathrm{~h}$; adherent and floating cells were then pelleted, washed with cold PBS and lysed in RIPA buffer [150 mM NaCl, 20 mM Tris- $\mathrm{HCl}$ (pH 7.5), $50 \mathrm{mM} \mathrm{NaF}, 20 \mu \mathrm{M}$ $\mathrm{Na}_{3} \mathrm{VO}_{4}$ and protease inhibitor]. Lysates were centrifuged at $15,000 \mathrm{rpm}$ at $4^{\circ} \mathrm{C}$ for $10 \mathrm{~min}$ and the protein concentration in each sample was determined by the BCA Protein Assay kit (Takara Bio, Inc., Shiga, Japan). Cell lysates or their fractions containing equal amounts of protein $(12 \mu \mathrm{g})$ were resolved by the Mini-Protean TGX Precast Gel (Bio-Rad Laboratories) and transferred to nitrocellulose membranes. Membranes were probed with the primary antibody, followed by the secondary antibody conjugated to HRP, developed using western blot detection reagents (Amersham Biosciences, Uppsala, Sweden), and then detected by the ChemiDoc SRS image analysis system (Bio-Rad Laboratories).

Statistical analysis. Statistical analyses were performed with IBM SPSS Statistics for Windows, version 21.0 (IBM Corp., Armonk, NY, USA). P-values of $<0.05$ were considered to indicate a statistically significant difference. We used Dunnett's test for the time-dependent cell growth assay. Data are presented as means \pm standard deviation.

\section{Results}

Determination of $I C_{50}$ for $C D D P$ and $17-A A G$ across a panel of ESCC cell lines. Treating esophageal cell lines with CDDP and 17-AAG resulted in dose-dependent cytotoxicity. Fig. 1 shows $\mathrm{IC}_{50}$ values for CDDP (Fig. 1A) and 17-AAG (Fig. 1B) across our panel of ESCC cell lines. $\mathrm{IC}_{50}$ values ranged from 0.983 to $14.9 \mu \mathrm{M}$ for CDDP, and 0.0128 to $2.37 \mu \mathrm{M}$ for 17-AAG, respectively. Based on these results, we decided to use KYSE30, KYSE150, EC-GI-10 and TE6 as representative CDDP-resistant cell lines in subsequent experiments.

$C D D P$ and 17-AAG exhibit synergistic inhibitory effects on the growth of KYSE30 and KYSE150 esophageal squamous carcinoma cell lines. To evaluate the impact of the combined treatment with CDDP and 17-AAG on CDDP-resistant cell 
Table I. Combination index value of the interaction between CDDP with 17-AAG against human esophageal carcinoma cell lines

\begin{tabular}{lccll}
\hline Cell line & $\begin{array}{c}\mathrm{IC}_{50} \text { of 17-AAG } \\
(\mu \mathrm{M})\end{array}$ & $\begin{array}{c}\text { Concentration of 17-AAG } \\
(\mu \mathrm{M})\end{array}$ & CI at $\mathrm{IC}_{50}$ & \multicolumn{1}{c}{ Interpretation } \\
\hline KYSE30 & 2.37 & 1.000 & 0.49 & \multicolumn{1}{c}{ Synergism } \\
& & 0.500 & 0.76 & Moderate synergism \\
KYSE150 & 0.10 & 0.050 & 0.71 & Moderate synergism \\
& \multirow{2}{*}{1.40} & 0.025 & 0.55 & Synergism \\
EC-GI-10 & \multirow{2}{*}{0.04} & 0.000 & 1.80 & Antagonism \\
& & 0.050 & 1.78 & Antagonism \\
TE6 & & 0.025 & 1.18 & Moderate antagonism \\
& & & 1.06 & Additive effect
\end{tabular}

Different concentrations of 17-AAG were employed to study the effect on $\mathrm{IC}_{50}$ of CDDP. Variable ratios of drug concentrations and mutually non-exclusive equations were used to determine the $\mathrm{CI}$. $\mathrm{CI}>1.3$ indicates antagonism; $\mathrm{CI}=1.1$ to 1.3 , moderate antagonism; $\mathrm{CI}=0.9$ to 1.1 , additive effect; $\mathrm{CI}=0.8$ to 0.9 , slight synergism; $\mathrm{CI}=0.6$ to 0.8 , moderate synergism; $\mathrm{CI}=0.4$ to 0.6 , synergism; and $\mathrm{CI}=0.2$ to 0.4 , strong synergism. CI, combination index; CDDP, cisplatin.
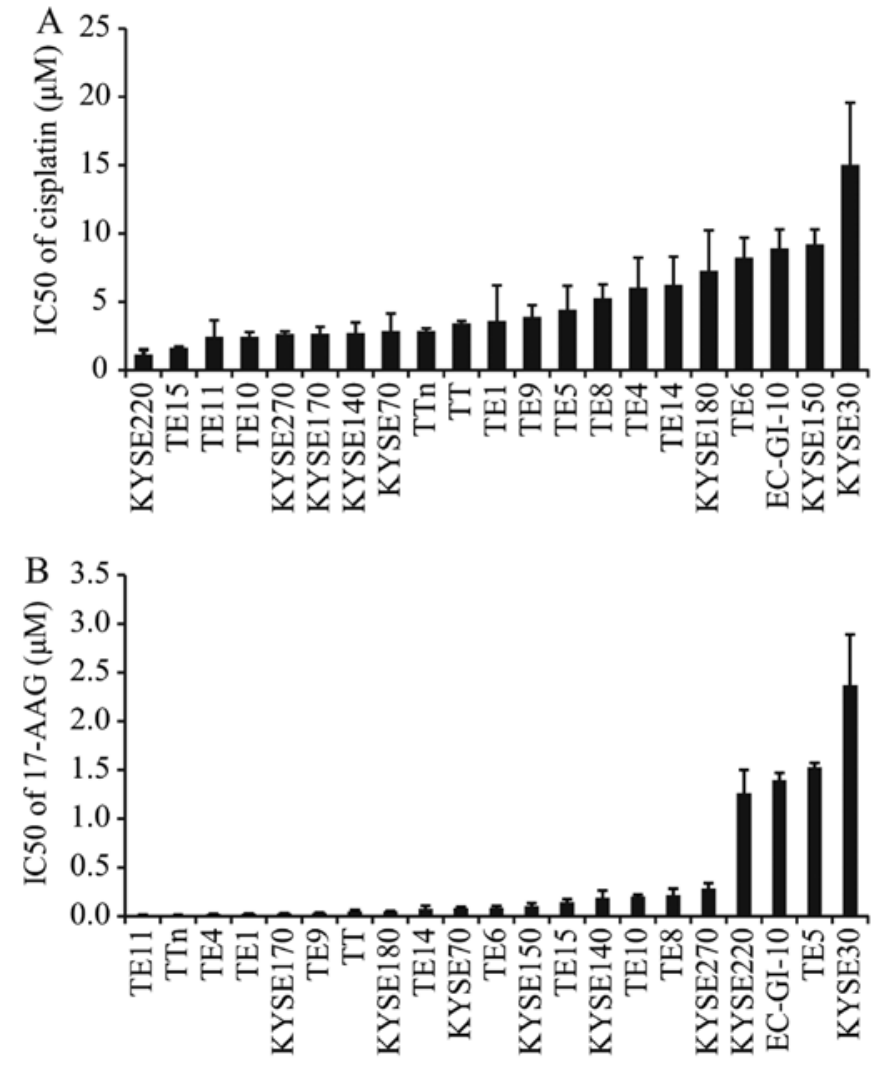

Figure 1. $\mathrm{IC}_{50}$ values for cisplatin (A) and 17-AAG (B) across the panel of esophageal squamous cell carcinoma cell lines. Cells were treated with a range of concentrations of cisplatin or 17-AAG for $72 \mathrm{~h}$. Viable cell density was determined by a water-soluble tetrazolium salt and $\mathrm{IC}_{50}$ was calculated. The values and error bars represent the mean and standard deviation of at least three experiments performed in quintuplicate.

lines, KYSE30, KYSE150, EC-GI-10 and TE6 were treated with various concentrations of each drug alone or in combination, and were then subjected to a cell viability assay. As shown in Fig. 2, the combination with low-dose 17-AAG shifted the survival curve to the left in KYSE30 and KYSE150. The
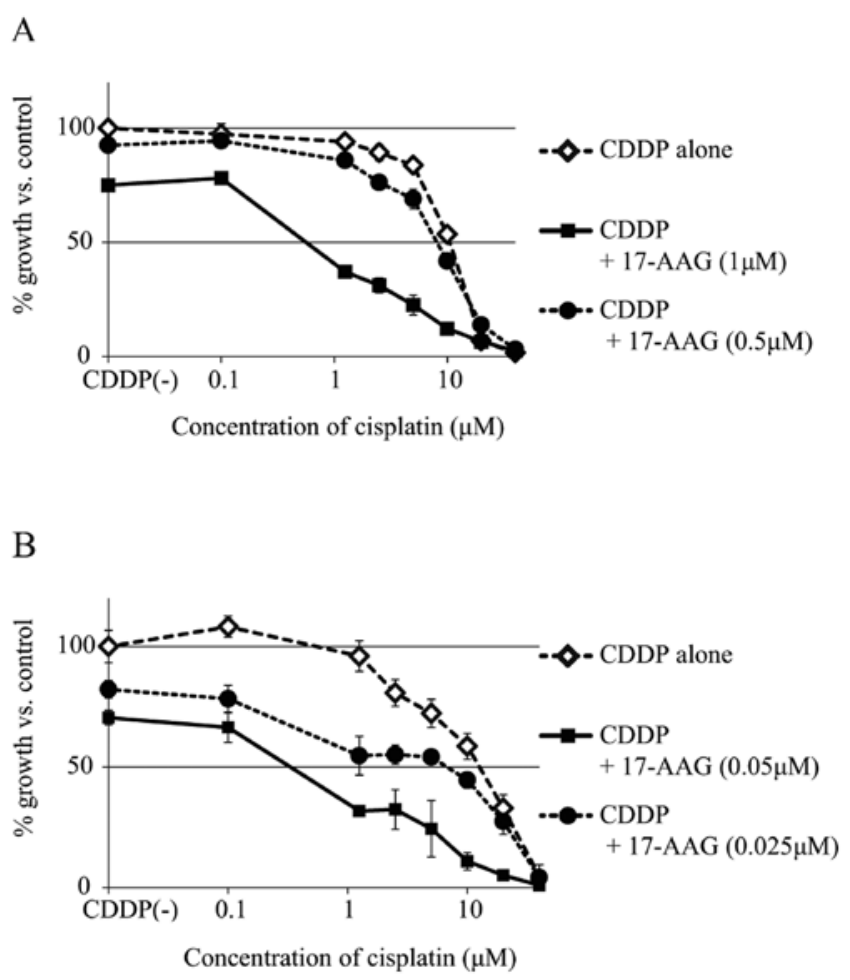

Figure 2. Dose-response curve for cisplatin (CDDP) in the presence of low doses of 17-AAG. KYSE30 and KYSE150 were treated with various doses of CDDP (0.1-40 $\mu \mathrm{M})$ and 17-AAG (0.5-1 $\mu \mathrm{M}$ for KYSE30; 0.025-0.05 $\mu \mathrm{M}$ for KYSE150) for $72 \mathrm{~h}$, and cytotoxicity was evaluated by a drug sensitivity assay. (A) KYSE30, (B) KYSE150. The experiment was performed twice and representative data are shown. Data are presented as means \pm standard deviation of quintuplicate wells.

interaction between CDDP and 17-AAG was determined by calculating the CI. The CI values of KYSE30 and KYSE150 ranged from 0.4 to 0.7 for $50 \%$ cell kill, demonstrating synergistic behavior between CDDP and 17-AAG (Table I). In contrast, the CI value of EC-GI-10 and TE6 ranged from 1.0 to 1.8. 

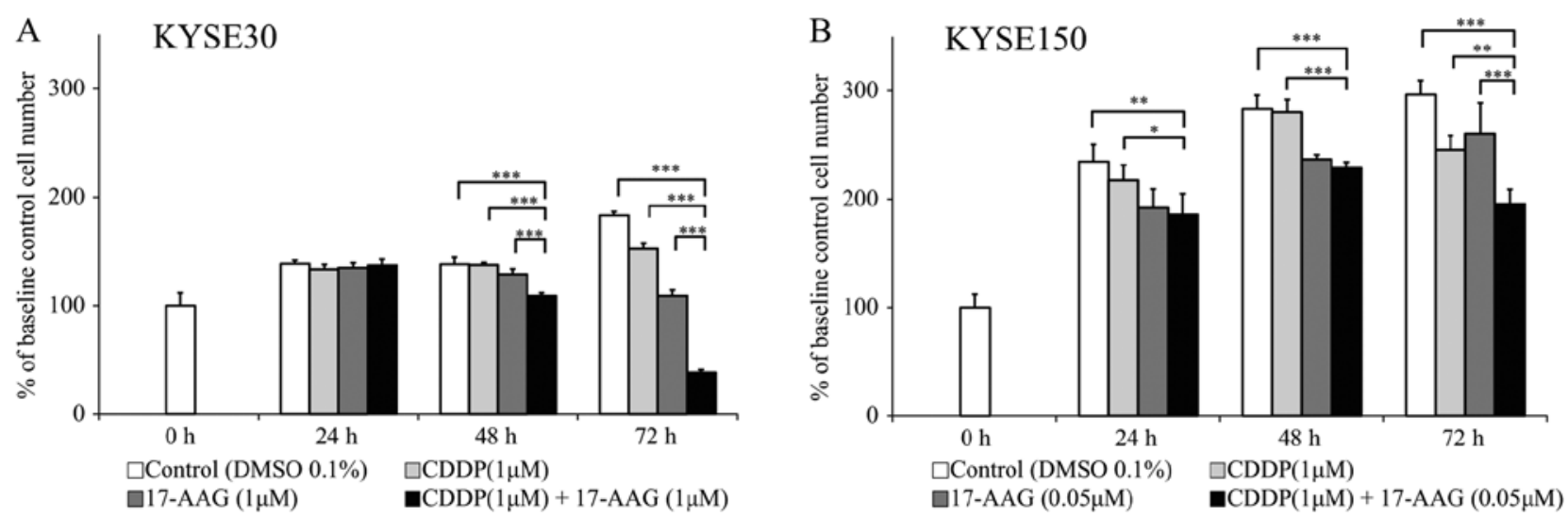

Figure 3. Changes in cell numbers by the treatment with cisplatin (CDDP) and 17-AAG, alone or in combination. (A) KYSE30 and (B) KYSE150 were exposed to 17-AAG alone ( $1 \mu \mathrm{M}$ for KYSE30, $0.05 \mu \mathrm{M}$ for KYSE150), CDDP alone ( $1 \mu \mathrm{M}$ for both cell lines), and the combination of CDDP (1 $\mu \mathrm{M}$ for both cell lines) and 17-AAG (1 $\mu \mathrm{M}$ for KYSE30, $0.05 \mu \mathrm{M}$ for KYSE150). Cell numbers were counted after 24, 48 and $72 \mathrm{~h}$. Data are presented as means and standard deviation of quintuplicate wells. ${ }^{*} \mathrm{P}<0.05,{ }^{, *} \mathrm{P}<0.01,{ }^{* * *} \mathrm{P}<0.001$.
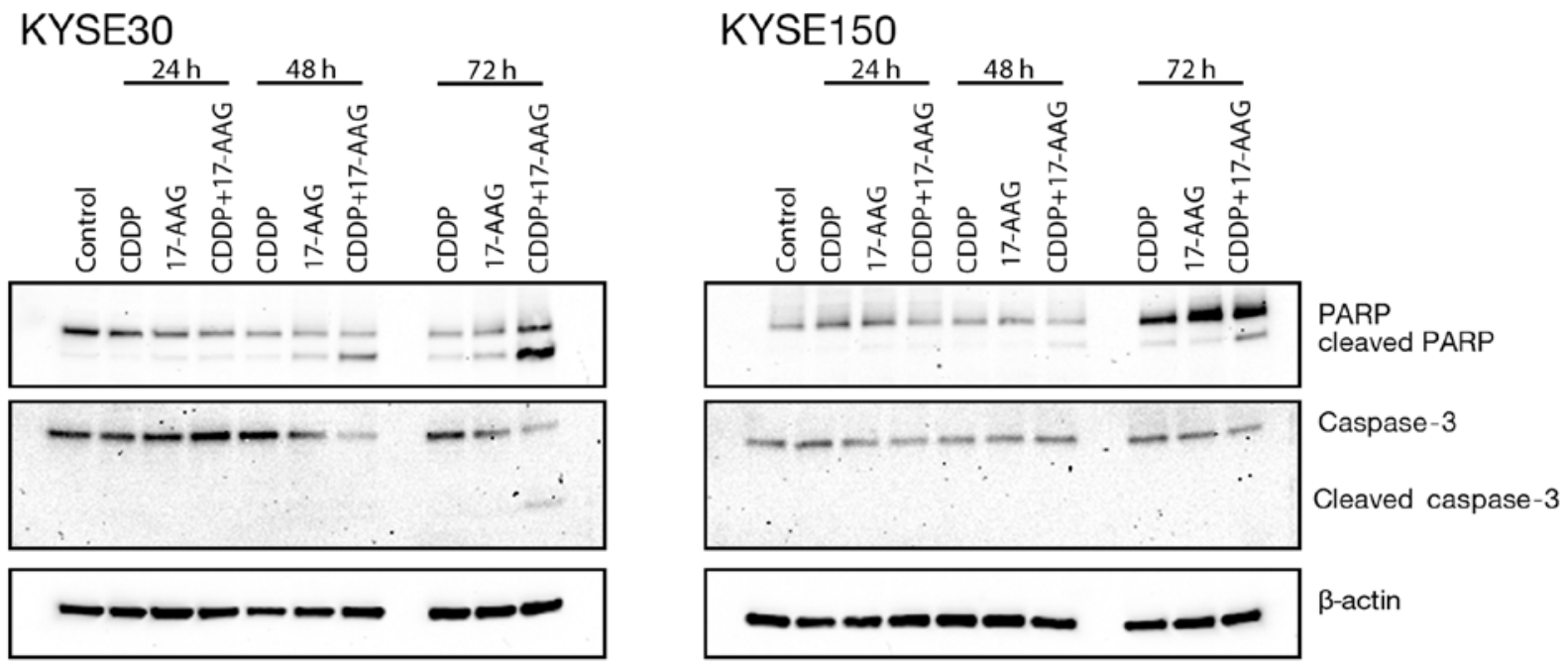

Figure 4. Induction of apoptosis by the combination of cisplatin (CDDP) and 17-AAG. KYSE30 was treated with CDDP (1 $\mu \mathrm{M})$ and/or 17-AAG (1 $\mu \mathrm{M})$, while KYSE150 was treated with CDDP $(1 \mu \mathrm{M})$ and/or 17-AAG $(0.05 \mu \mathrm{M})$. After the treatment, cell extracts were examined by western blot analysis.

These results showed either the additive or antagonistic effects of CDDP and 17-AAG in EC-GI-10 and TE6.

Cell count assay. To confirm the synergistic effect of CDDP and 17-AAG in KYSE30 and KYSE150, we counted actual cell numbers after treatment with the vehicle only $(0.1 \%$ DMSO), $1 \mu \mathrm{M}$ CDDP only, $1 \mu \mathrm{M}$ 17-AAG only, and the combined treatment with $1 \mu \mathrm{M}$ CDDP and $1 \mu \mathrm{M} 17-\mathrm{AAG}$ for 24,48 and $72 \mathrm{~h}$.

As shown in Fig. 3, low-dose CDDP alone and low-dose 17-AAG alone modestly suppressed cell growth in KYSE30 and KYSE150 (Fig. 3). However, the reduction in cell growth was significantly greater with the combination of CDDP and 17-AAG than with either drug alone.

Combination of CDDP and 17-AAG induces apoptosis. Using western blot analysis, we determined whether the reduced cell growth caused by the combined treatment with CDDP and 17-AAG occurred due to the induction of apoptosis via the cleavage of poly (ADP-ribose) polymerase (PARP) and activation of caspase-3.
No significant changes were observed in the expression of PARP and cleaved PARP $24 \mathrm{~h}$ after treatment with CDDP alone, 17-AAG alone, or CDDP and 17-AAG (Fig. 4). However, a clear increase was observed in cleaved PARP $48 \mathrm{~h}$ after the co-treatment with CDDP and 17-AAG in KYSE30 and KYSE150. The increase in cleaved PARP was greater than that with either drug alone. The induction of cleaved PARP was further enhanced at $72 \mathrm{~h}$. Cleaved caspase- 3 was detected $72 \mathrm{~h}$ after the co-treatment with CDDP and 17-AAG in KYSE30, but the band was weaker than that of cleaved PARP.

Combination of CDDP and 17-AAG reduces the expression of XIAP and phosphorylated Akt. To understand the mechanism underlying the synergy between CDDP and 17-AAG, we investigated the expression of proteins associated with apoptosis (Fig. 5). Under basal conditions, KYSE30 expressed high levels of XIAP, cIAP1, and survivin and low levels of cIAP2. KYSE150 expressed high levels of XIAP and cIAP1, and low levels of cIAP2 and survivin. Livin was not detected in either cell line. 


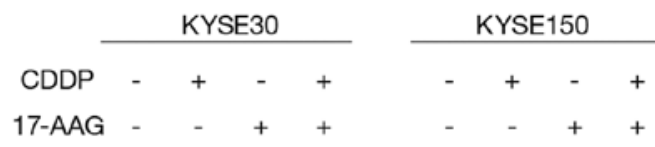

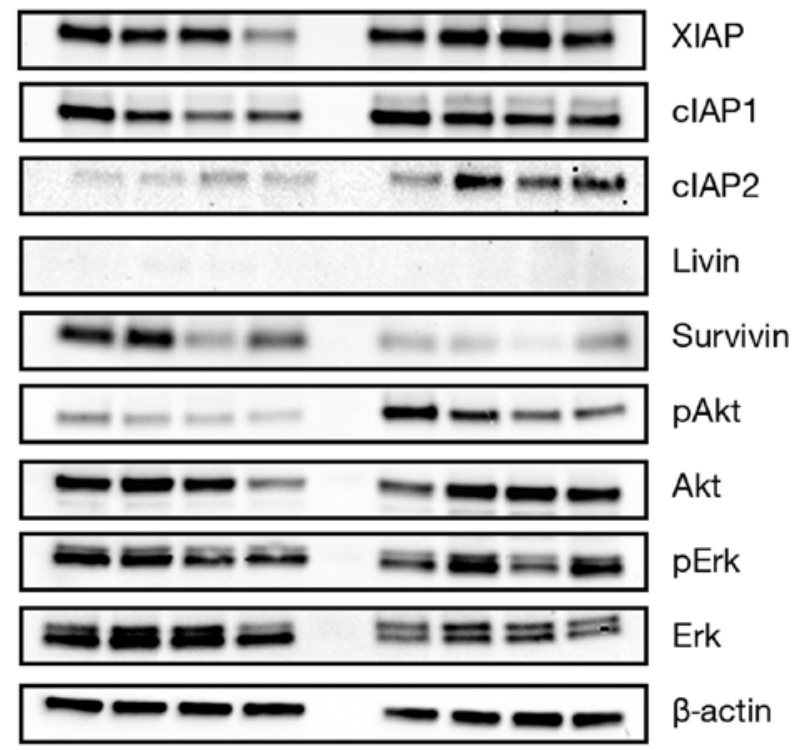

Figure 5. Western blot analysis of the IAP family proteins, the Erk and Akt pathways. KYSE30 and KYSE150 were treated with cisplatin (CDDP; $1 \mu \mathrm{M}$ ), 17-AAG ( $1 \mu \mathrm{M}$ for KYSE30, $0.05 \mu \mathrm{M}$ for KYSE150), and the combination of these drugs for $72 \mathrm{~h}$, and cell lysates were analyzed by western blotting.

The treatment with CDDP alone, 17-AAG alone, or combined treatment with CDDP and 17-AAG induced variable changes in the levels of cIAP1, cIAP2 and survivin; however, a correlation was not observed between these changes and cell growth inhibition or the induction of apoptosis by the treatment. XIAP levels were, in contrast, unchanged by either agent alone, but were significantly reduced by the combined treatment. We also examined the expression of Bcl-2 family members, including Bcl-2, Bcl-xL, Bid, Bad, Bax, Bim, Beclin and BAG-1; however, no significant changes were observed by the treatment with CDDP alone, 17-AAG alone, or combined treatment with CDDP and 17-AAG (data not shown). These results demonstrated that the combination of CDDP and 17-AAG mainly induced apoptosis by inhibiting XIAP.

To identify the mechanism for the reduction in XIAP, the expression of the Akt and the Erk pathways was examined. As shown in Fig. 5, phosphorylated Akt levels were slightly reduced by either CDDP or 17-AAG alone in both cell lines. However, the combination of CDDP and 17-AAG clearly diminished phosphorylated Akt levels (Fig. 5). Phosphorylated Erk levels remained unchanged by the treatment in KYSE30. Phosphorylated Erk levels were modestly increased by CDDP alone and the combined treatment with CDDP and 17-AAG in KYSE150; however, no correlation was observed between these changes and the inhibition of cell growth or induction of apoptosis.

Time-dependent changes in the expression of phosphorylated $A k t$, total Akt, and XIAP. In order to determine time-dependent changes in phosphorylated Akt, total Akt and XIAP, we examined the expression of these proteins after the treatment with CDDP and/or 17-AAG, either alone or in combination (Fig. 6). Phosphorylated Akt levels were modestly reduced by 17-AAG alone and by the combination of CDDP and 17-AAG after $24 \mathrm{~h}$. Further reductions occurred at $72 \mathrm{~h}$, especially with the combination of CDDP and 17-AAG in KYSE30. The expression of phosphorylated Akt was not significantly changed by CDDP alone. The expression of XIAP was reduced by 17-AAG alone and by the combination of CDDP and 17-AAG, similar to that for phosphorylated Akt.

\section{Discussion}

In the present study, we demonstrated that the combined treatment with CDDP and 17-AAG had synergistic inhibitory effects on cell growth in CDDP-resistant ESCCs. The synergistic interaction between CDDP and 17-AAG resulted in significant increases in the cytotoxicity of CDDP; a strong cytotoxic effect was obtained in the presence of low-dose 17-AAG, which hardly has a cytotoxic effect by itself, in combination with a low concentration of CDDP. This cytotoxic effect occurred via induction of apoptosis, as demonstrated by the cleavage of PARP and caspase- 3 .

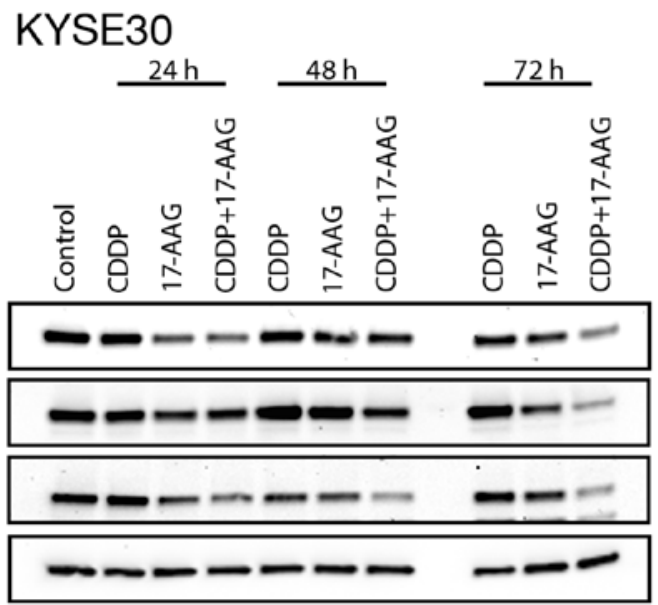

\section{KYSE150}

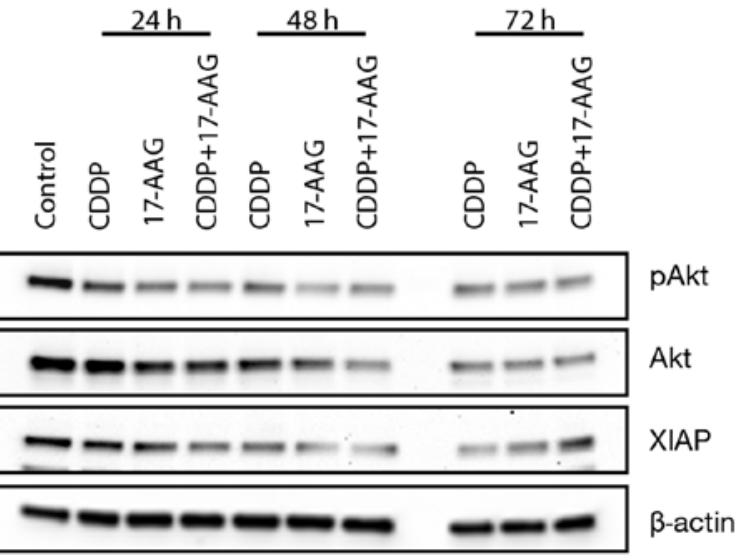

Figure 6. Time-dependent changes in the expression of phosphorylated Akt, Akt and XIAP. KYSE30 and KYSE150 were treated with cisplatin (CDDP; $1 \mu \mathrm{M})$ 17-AAG ( $1 \mu \mathrm{M}$ for KYSE30, $0.05 \mu \mathrm{M}$ for KYSE150), and the combination of these drugs for the indicated times, and cell lysates were analyzed by western blotting. 
The synergy between CDDP and an HSP90 inhibitor has been reported in previous studies; for example, McCollum et al reported that CDDP was synergistic with geldanamycin or 17-AAG and combined treatment with the two drugs increased apoptosis in the lung cancer cell line A549 (12). This synergy was attributed in part to the CDDP-induced abrogation of heat shock factor-1 activity. Weng et al also showed that 17-AAG enhanced CDDP cytotoxicity in the non-small cell lung cancer cell lines, A549 and H1650 (13). This synergistic effect was reported to be mediated by the downregulation of thymidine phosphorylase and Akt activation. We have yet to examine whether similar mechanisms occur in our cell lines. Nevertheless, our study provides the first evidence for the synergy of CDDP and 17-AAG in CDDP-resistant esophageal squamous cell lines.

We also revealed that, among the major regulators of apoptosis, the Akt/XIAP pathway mediated the synergistic effect of CDDP and 17-AAG; the expression patterns of phosphorylated Akt and XIAP levels closely correlated with their inhibitory effect on cell growth, induction of PARP cleavage, and apoptosis, which occurred by the combined treatment with CDDP and 17-AAG. Moreover, time-course experiments demonstrated that the reduction in phosphorylated Akt and XIAP levels were concomitant with the induction of PARP cleavage and apoptosis.

Previous studies indicated the Akt/XIAP pathway as the main regulator of apoptosis by chemotherapy in some cancer cell lines, including carcinomas of the breast, ovary, uterine cervix and melanoma (14-17). Akt phosphorylates and stabilizes XIAP, and the deactivation or knockdown of Akt destabilizes XIAP, leading to apoptosis (18). In addition, specific inhibition of XIAP expression was shown to induce apoptosis and increase caspase-3 activity in prostate cancer cells (19). These findings support our conclusion that the synergistic effect of CDDP and 17-AAG is mediated by the induction of apoptosis via the Akt and XIAP pathways.

One limitation of our study is that we lack data showing the synergy of CDDP and 17-AAG in vivo, and such data will be required to extrapolate the current results to clinical situations. We also cannot explain why synergy occurred in KYSE30 and KYSE150, but not in EC-GI-10 or TE6. A recent study indicated phosphatase and tensin homolog (PTEN) as a potential predictive marker for HSP90 inhibitor sensitivity among four ESCC cell lines (20). However, biomarkers that predict the HSP90 response or synergy of an HSP90 inhibitor and CDDP have not yet been established (21). The identification of such biomarkers is crucial in clinical settings.

In conclusion, we showed that the combination of low concentrations of CDDP with low-dose 17-AAG exerted synergistic effects on CDDP-resistant ESCC cell lines. The mechanism of the synergy was attributed to apoptosis mediated by downregulation of the Akt/XIAP pathway. Our results indicate that the co-administration of low-dose 17-AAG and CDDP overcomes CDDP chemoresistance and may improve the outcomes of patients with ESCC.

\section{References}

1. Jemal A, Bray F, Center MM, Ferlay J, Ward E and Forman D: Global cancer statistics. CA Cancer J Clin 61: 69-90, 2011.

2. Ozawa S, Tachimori Y, Baba H, et al: Comprehensive registry of esophageal cancer in Japan, 2004. Esophagus 9: 75-98, 2012.

3. Miyata H, Yamasaki M, Kurokawa Y, et al: Multimodal treatment for resectable esophageal cancer. Gen Thorac Cardiovasc Surg 59: 461-466, 2011.

4. Berrino F, De Angelis R, Sant M, et al: Survival for eight major cancers and all cancers combined for European adults diagnosed in 1995-99: results of the EUROCARE-4 study. Lancet Oncol 8: 773-783, 2007.

5. Siegel R, Naishadham D and Jemal A: Cancer statistics, 2012. CA Cancer J Clin 62: 10-29, 2012.

6. Yamasaki M, Miyata H, Tanaka K, et al: Multicenter phase I/II study of docetaxel, cisplatin and fluorouracil combination chemotherapy in patients with advanced or recurrent squamous cell carcinoma of the esophagus. Oncology 80: 307-313, 2011.

7. Hara H, Tahara M, Daiko H, et al: Phase II feasibility study of preoperative chemotherapy with docetaxel, cisplatin, and fluorouracil for esophageal squamous cell carcinoma. Cancer Sci: Aug 30, 2013 (Epub ahead of print).

8. Neckers L and Neckers K: Heat-shock protein 90 inhibitors as novel cancer chemotherapeutic agents. Expert Opin Emerg Drugs 7: 277-288, 2002.

9. Newman B, Liu Y, Lee HF, Sun DX and Wang Y: HSP90 inhibitor 17-AAG selectively eradicates lymphoma stem cells. Cancer Res 72: 4551-4561, 2012.

10. Zhang $\mathrm{H}$ and Burrows F: Targeting multiple signal transduction pathways through inhibition of Hsp90. J Mol Med 82: 488-499, 2004.

11. Chou TC: Theoretical basis, experimental design, and computerized simulation of synergism and antagonism in drug combination studies. Pharmacol Rev 58: 621-681, 2006.

12. McCollum AK, Lukasiewicz KB, Teneyck CJ, Lingle WL, Toft DO and Erlichman C: Cisplatin abrogates the geldanamycininduced heat shock response. Mol Cancer Ther 7: 3256-3264, 2008.

13. Weng SH, Tseng SC, Huang YC, Chen HJ and Lin YW: Inhibition of thymidine phosphorylase expression by using an HSP90 inhibitor potentiates the cytotoxic effect of cisplatin in non-small-cell lung cancer cells. Biochem Pharmacol 84: 126-136, 2012.

14. Pramanik KC, Kudugunti SK, Fofaria NM, Moridani MY and Srivastava SK: Caffeic acid phenethyl ester suppresses melanoma tumor growth by inhibiting PI3K/AKT/XIAP pathway. Carcinogenesis 34: 2061-2070, 2013.

15. Rajput S, Kumar BN, Sarkar S, et al: Targeted apoptotic effects of thymoquinone and tamoxifen on XIAP mediated Akt regulation in breast cancer. PLoS One 8: e61342, 2013.

16. Gagnon V, Van Themsche C, Turner S, Leblanc V and Asselin E: Akt and XIAP regulate the sensitivity of human uterine cancer cells to cisplatin, doxorubicin and taxol. Apoptosis 13: 259-271, 2008.

17. Asselin E, Mills GB and Tsang BK: XIAP regulates Akt activity and caspase-3-dependent cleavage during cisplatin-induced apoptosis in human ovarian epithelial cancer cells. Cancer Res 61: 1862-1868, 2001.

18. Dan HC, Sun M, Kaneko S, et al: Akt phosphorylation and stabilization of X-linked inhibitor of apoptosis protein (XIAP). J Biol Chem 279: 5405-5412, 2004.

19. Amantana A, London CA, Iversen PL and Devi GR: X-linked inhibitor of apoptosis protein inhibition induces apoptosis and enhances chemotherapy sensitivity in human prostate cancer cells. Mol Cancer Ther 3: 699-707, 2004.

20. Bao XH, Takaoka M, Hao HF, et al: Antiproliferative effect of the HSP90 inhibitor NVP-AUY922 is determined by the expression of PTEN in esophageal cancer. Oncol Rep 29: 45-50, 2013.

21. Garcia-Carbonero R, Carnero A and Paz-Ares L: Inhibition of HSP90 molecular chaperones: moving into the clinic. Lancet Oncol 14: e358-e369, 2013. 